\title{
HST and spectroscopic observations of the L1551 IRS5 jets (HH154) ${ }^{\star}$
}

\author{
C. V. M. Fridlund ${ }^{1}$, R. Liseau ${ }^{2}$, A. A. Djupvik ${ }^{3}$, M. Huldtgren ${ }^{2}$, G. J. White ${ }^{2,4}$, F. Favata ${ }^{1}$, and G. Giardino ${ }^{1}$ \\ ${ }^{1}$ ESA Astrophysics Missions Division, Research and Scientific Support Department, ESTEC, PO Box 299, 2200 AG, \\ Noordwijk, The Netherlands \\ e-mail: malcolm.fridlund@esa.int \\ 2 Stockholm Observatory, SCFAB, Roslagstullsbacken 21, 10691 Stockholm, Sweden \\ 3 Nordic Optical Telescope, Apartado 474, 38700 Santa Cruz de La Palma Canarias, Spain \\ ${ }^{4}$ Center for Astrophysics and Planetary Science, University of Kent, Canterbury, Kent, CT2 7NZ, UK
}

Received 22 November 2004 / Accepted 16 March 2005

\begin{abstract}
We have carried out a thorough optical study of the closest star formation jet. The inner arcminute surrounding the class 0/I binary protostar L1551 IRS5 and its associated jet (HH154) have been observed using the Hubble Space Telescope with the WFPC2 camera, the ESO New Technology Telescope with the EMMI spectrograph and the Nordic Optical Telescope with the ALFOSC spectrograph. This data set is compared to earlier ground based imaging with the aim to study the evolution of this particular jet, and its possible interaction with the molecular material in the bipolar molecular outflow associated with this source. The velocity field of the jet is mapped out. The highest velocities are found in the vicinity of the recently discovered $\mathrm{X}$-ray source emanating from a shock in this jet. The energy radiated by the X-ray source is compatible with these velocities. The $\mathrm{H} \alpha$ and $\mathrm{H} \beta$ emission from the jet is used to determine the extinction, which is found to increase inwards in the jet towards the protostar. The extinction towards the X-ray source is consistent with the one determined from the X-ray spectrum.
\end{abstract}

Key words. ISM: individual objects: L1551 - ISM: jets and outflows - ISM: Herbig Haro objects - stars: formation stars: pre-main sequence

\section{Introduction}

Among bipolar molecular outflows, the one centered on the young stellar object IRS5 in L1551 was the first discovered (Snell et al. 1980). It is relatively nearby, $\sim 150 \mathrm{pc}$ (Kenyon et al. 1994; Reipurth 1999), allowing the study of small spatial elements, and oriented with its major outflow axis most likely at an angle of between $45^{\circ}$ and $60^{\circ}$ (Liseau et al. 2005) with respect to the plane of the sky. This results in red- and blueshifted CO outflow lobes which are spatially well separated, and which have an angular extent of several tens of arcminutes on the sky. The red- and the blue-shifted CO lobes contains several Herbig Haro objects (HH objects) most prominently seen in the blue-shifted lobe, (e.g. HH28, HH29). In Cudworth \& Herbig (1979) and Fridlund et al. $(1984,1993,1998)$ the

\footnotetext{
* Based on observations made with the Nordic Optical Telescope, operated on the island of La Palma jointly by Denmark, Finland, Iceland, Norway, and Sweden, in the Spanish Observatorio del Roque de los Muchachos of the Instituto de Astrofisica de Canarias, and based on observations made with the NASA/ESA Hubble Space Telescope, obtained at the Space Telescope Science Institute, which is operated by the Association of Universities for Research in Astronomy, Inc., under NASA contract NAS5-26555.
}

authors argue, based on proper motion vectors, velocity gradients in $\mathrm{CO}$, and the radial velocity field, that the outflow is originating at IRS5 and is interacting with the ambient medium at the postion of the Herbig Haro objects. In Devine et al. (1999, 2000), the authors on the other hand argue that the HH28 and HH29 are excited by a flow from another YSO, i.e. L1551NE.

The low mass characteristics of IRS5 were determined by observations in the far infrared (Fridlund et al. 1980). A jet has been observed at optical wavelengths (Mundt \& Fried 1983; Neckel \& Staude 1987; Fridlund \& Liseau 1994, 1998), in the near IR (Campbell et al. 1988; Pyo et al. 2002, 2005) and in the radio continuum (Cohen et al. 1982; Rodríguez et al. 1986). The realisation that this jet has Herbig Haro characteristics (Sarcander et al. 1985) has led to it receiving the designation HH154 in the compilation of Reipurth (1999). Morphological changes were detected within the jet by Sarcander et al. (1985) and Neckel \& Staude (1987). The latter found that over the epoch 1983-1987, a new knot appeared near IRS5, and that the transverse velocities for several knots in the jet were of order $\sim 190 \mathrm{~km} \mathrm{~s}^{-1}$ - in agreement with earlier proper motion measurements of one of the knots by Sarcander et al. (1985). Neckel \& Staude (1987) introduced a consistent nomenclature 
for the features observed along the jet. This was also used by Fridlund \& Liseau (1994) - hereafter FL94 and Fridlund \& Liseau (1998) - hereafter FL98, and we continue to use their designation in this paper, consistently updated in view of the evolution of the jet.

At all observed wavelengths, the jet is relatively compact compared to the molecular outflow, growing from $\sim 7$ arcsec to $\sim 14$ arcsec between 1983 and 2001. The jet terminates in a bright emission knot - "D" according to the nomenclature described above - although some fainter, isolated emission peaks (A, B and C) are also detected further "downstream". Mundt et al. (1991) used image restoration techniques on a deep [S II] image and suggested that the jet consists of a limb brightened cavity, with a high velocity wind permeating the cavity, which through interactions with the walls of the cavity, produce the HH knots. The optical properties of the jet were then further elucidated by FL94 who reported on high spatial resolution monitoring observations of the L1551 IRS5 jet carried out between 1989 and 1993, made from the ground in excellent seeing conditions. FL94 confirmed the earlier results by Neckel \& Staude (1987) that morphological changes along the jet have dynamical time-scales of $<$ a few years, and that these changes can be interpreted as proper motions of knots within the jet. Disappearance of "old" and appearance of "new" knots was recorded. It was also found that the brightest feature (D) in the jet accounts for more than $50 \%$ of the observed total emission in the lines of $\mathrm{H} \alpha$ and [S II] 6716, $6731 \AA$. Also from these observations, three different transversal velocity components $\left(120 \mathrm{~km} \mathrm{~s}^{-1}, 190 \mathrm{~km} \mathrm{~s}^{-1}\right.$ and $>310 \mathrm{~km} \mathrm{~s}^{-1}$ ) were identified. Taken together with earlier radial velocity measurements found in the literature, FL94 hypothesized that the spatial distribution of the knots was best represented by the features moving close to the surface of a conical volume. FL98, which reports preliminary results from the first visit in the two-epoch Hubble Space Telescope (HST) program fully described in the current paper, interprets their data together with some spectroscopic data as indicative of the existence of two separate jet-flows originating from IRS5. Each of these is possibly originating from one of the two components of IRS5 found by Looney et al. (1997). FL98 also identified the brightest component ("D") of the jet with the "working surface" of the "northern jet" and discussed the structure of that shock. Using the models of Hartigan (1989), they found that the ionisation fraction appears high ( $~ 50 \%-100 \%)$. From the velocities determined, an estimate of the forces required to accelerate the individual knots could be made. This was compared to the force required to accelerate the molecular gas in the same volume of space as the jet (Fridlund $\&$ Knee 1993) and found to be at least two orders of magnitude too low assuming an ionisation fraction of $\sim$ unity. FL98 pointed out the impact of this result on the question of the level of ionisation in optical jets which has turned into a major problem when trying to unify models for optically visible jets with those of the accompanying molecular outflows.

The structures closest to IRS5 have been observed with interferometers in the radio continuum $(1.4 \mathrm{GHz}$ to $\sim 220 \mathrm{GHz})$, by a number of researchers (Bieging \& Cohen 1985; Snell et al. 1985; Rodríguez et al. 1986; Keene \& Masson 1990; Ladd et al. 1995; Looney et al. 1997). The latter authors pointed out that a 3 component model emerges with a large scale (10 to 30 arcsec) envelope, a disk like feature ( $\sim 1$ arcsec $)$ and a binary source with individual components separated by 0.35 arcsec, and where the disks have each a $F W H M$ of $\lesssim 0.3$ arcsec. This double source, which was originally discovered by Bieging \& Cohen (1985) and at that time interpreted by Rodríguez et al. (1986) as the inner ionized edges of a gas and dust toroid, is now seen as a young binary system (also suggested by Bieging $\&$ Cohen 1985) with a separation of 50 AU (Looney et al. 1997). Rodríguez et al. (2003a,b) have analysed interferometric radio continuum observations, and trace the two jets to their origin. Based on that data, those authors suggest that the northern jet-component emanates from the southern binary and vice versa. It has also been demonstrated (Fridlund et al. 2002; White et al. 2000) that there is also a fourth component in the form of a large $(\sim 15000 \mathrm{AU})$, massive (several $\left.M_{\odot}\right)$, rotating flattened envelope of molecular gas.

In the L1551 cloud, we find the astrophysical jets located nearest to the Earth (HH154, HH30 and the HL Tau jet). This implies that for HH154, the HST can achieve similar $\left(\sim 10^{14} \mathrm{~cm}\right)$, or better, spatial resolution to that used in current 2D hydro-dynamical calculations of jets (Raga et al. 2004). A multi-cycle observing programme with the HST consequently appeared well motivated, with the primary goal being to study the time evolution of the jet, to use the shock diagnostic emission lines of $\mathrm{H} \alpha$ and the [S II] doublet at $\lambda \lambda 6717,6731 \AA$ in order to determine its physical properties. To detect fainter details we also obtained $R$-band ( $F 675 W$ filter) observations. Further we wished to study the structure furthest in towards IRS5. Since the jet here suddenly appears from behind $\sim 100$ mag of obscuring material (presumably the accretion disk), we try to observe light scattered up along the major axis of the jet. To achieve this we selected the F814W I-band filter. As mentioned above, preliminary results have been reported in Fridlund et al. (1997) and FL98. Included in the latter paper are some (also preliminary) ground based spectroscopic data, demonstrating a radial velocity gradient along the jet, as well as two separate velocity fields.

Recently, X-rays emanating from the jet have been discovered (Favata et al. 2002). The observations carried out with XMM-Newton have a positional uncertainty of roughly the same size as the jet. The X-ray source with a temperature of a few million degrees and a visual extinction of $\sim 5$ mag, was tentatively identified as originating from the working surface ("knot D"), mainly because of its brightness, and feature D being the only source with reported [O III] emission then known (Cohen \& Fuller 1985; Sarcander et al. 1985). The X-rays had to originate in the jet, since the star itself is hidden by the very large extinction from the surrounding disk. The position of the X-ray source have recently been measured by Bally et al. (2003), who observed with the Chandra X-ray observatory providing a spatial resolution of $\sim 1$ arcsec. This has enabled them to determine that the X-rays emanate roughly from $\sim 0.5 \operatorname{arcsec}$ to 1 arcsec from the radio position of IRS5.

In the present paper we give the detailed results and interpretation from two HST epochs (cycle $5 \&$ cycle 6 ). We also use new, extensive ground based imaging and spectroscopic data to support our interpretation. 
We confirm the dual structure of the jet itself, and we describe the somewhat surprising morphology found close to the base of the jet. The time evolution of the jet is described, as discerned from the measured proper motions of individual knots within the jet. We also discuss the structure of different individual shocks, including the excitation conditions and the electron density along the jet and within separate knots, identify the velocity component associated with the X-ray source and the implications of having a strong X-ray source illuminating the accretion disk surrounding IRS5.

\section{Observations and data reduction}

\subsection{HST Imaging}

The area surrounding the L1551 IRS5 jet was imaged using the Wide Field and Planetary Camera 2 (WFPC2 - Trauger et al. 1994) aboard the Hubble Space Telescope. This camera consists of 4 adjacent $800 \times 800$ Loral CCD's. Three of these arrays have an image scale of $0.1 \operatorname{arcsec~pix}^{-1}$ (WF-chips), while the fourth - the planetary camera (PC-chip) - has an image scale of 0.046 arcsec pix $^{-1}$, thus well sampling the diffraction pattern in the $R$ - and $I$-band. The FOV in the planetary camera is $25^{\prime \prime} \times 25^{\prime \prime}$ and since the jet visible in the ground imagery is less than 15 arcsec long, we centered the PC on the position of IRS5. We used an orientation that allowed us to detect the only visible star within the field-of-view on one of the WF chips, leading to potential relative astrometry between epochs of $\sim 0.1$ arcsec. With this orientation we unfortunately could not image any of the other brighter nebulosities associated with L1551 IRS5. Note that the brightest object, HH29, has been imaged by Devine et al. (2000). Some fainter nebulosities are, however, visible on one of the WF chips. In this paper we only discuss features relevant to the jet.

We observed HH154 during 3 HST visits. During each visit, two exposures each were obtained through the F656N $(\mathrm{H} \alpha)$ and the $F 673 N(\lambda 6717 \AA+6731 \AA)$ filters beginning on 1996.034. Total exposure time for each filter was 2500 s. On 1996.078 we obtained 3 exposures each through the $F 675 W(R)$ and $F 814 W(I)$ filters with the total observing times being $1800 \mathrm{~s}$ and $2400 \mathrm{~s}$ respectively. We again obtained data through the $F 656 N(\mathrm{H} \alpha)$ and $F 673 N(\lambda 6717 \AA+6731 \AA)$ filters on 1998.074, providing us with a time base of almost exactly two years (727 days).

The data were reduced and calibrated into units of erg $\mathrm{cm}^{-2} \mathrm{~s}^{-1}$ using standard methods as described in e.g. Holtzman et al. (1995), Heathcote et al. (1996), Reipurth et al. (1997) and the WFPC2 handbook.

All the images were registered with each other by using the stellar image of VSS4 and the rotation angle information. The accuracy in this process is estimated to be $\sim 0.1 \mathrm{arcsec}$.

\subsection{Ground based imaging}

In December 1996 and in October 2001, calibrated images were obtained using the Nordic Optical Telescope. The instrument combination was identical to that used in the ground based spectroscopy except that ALFOSC was set to imaging mode. Images were obtained through a number of filters, of which results for $\mathrm{H} \alpha, \mathrm{H} \beta,\left[\mathrm{S}_{\mathrm{II}}\right] 4067,68 \AA$ and [O III] $5007 \AA$ are reported here, while a more detailed description, as well as the presentation of further data is deferred to a later paper.

\subsection{High resolution spectroscopy}

Spectroscopic observations of the jet were carried out with the NTT 3.58 m telescope - EMMI (ESO Multi-Mode Instrument) combination at the European Southern Observatory, La Silla, Chile. The observation was carried out on the night of 22 December 1998. We used the echelle mode with grating No. 10 and grism No. 6, resulting in a wavelength coverage of $6000 \AA-8400 \AA$. Using a $1^{\prime \prime} \times 10^{\prime \prime}$ slit, we have a spectral resolution of 28000 (equivalent to a velocity resolution of $\sim 11 \mathrm{~km} \mathrm{~s}^{-1}$ at $\mathrm{H} \alpha$ ). The seeing was about 1 arcsec, and three slit positions, each separated by the same amount in declination, were observed. These observations only cover the working surface of the jet (feature D). Lines detected with the measured fluxes and velocities are listed in Table 3. The reduction of the data were carried out with the ECHELLE context within the ESO MIDAS reduction package, and with flux calibration being obtained by observing the flux standard HR1544 (see http://www. hq. eso.org/observing/standards/spectra for details).

\subsection{Medium resolution spectroscopy}

The second set of spectroscopic observations was obtained at the Nordic Optical Telescope $(2.6 \mathrm{~m})$ in La Palma on the nights of 28 and 29 December 1999. We used the ALFOSC imager/spectrograph. This instrument was used in echelle mode and was equipped with a Loral $2 \mathrm{k} \times 2 \mathrm{k}$ CCD with $15 \mu \mathrm{m}$ pixels corresponding to $0.189 \mathrm{arcsec}_{\mathrm{pixel}}{ }^{-1}$. The slit was $13.8 \mathrm{arcsec}$ long, but due to crowding of the higher orders, only $12 \operatorname{arcsec}$ has been used. The slit width utilized was 1 arcsec. The grating/grism combination has a practical wavelength coverage of $4800 \AA-11000 \AA$, with a spectral resolution of 4300 resulting in a velocity resolution of $\sim 70 \mathrm{~km} \mathrm{~s}^{-1}$ at $\mathrm{H} \alpha$. Special care was taken in achieving precision pointing of the slit on the sky. Thus a short (20 s) exposure through an $R$-filter was obtained at each position. Then without moving the telescope, an image of the slit was made on the detector. Finally, the spectrum was recorded followed by another slit image and $R$-band image before moving to the next postion. This allows us to spatially register our spectra to within one pixel $(0.189 \mathrm{arcsec})$. A total of four (4) positions was recorded, with an integration time of $3600 \mathrm{~s}$ per position. The positions of the slits on the sky can be seen in Fig. 8. Also here the reductions of the data were carried out with the ECHELLE context within the ESO MIDAS reduction package, and with flux calibration being obtained by observing the two flux standards hz4 and BD+28 4211 (see http://www. hq. eso.org/observing/standards/spectra for details). 

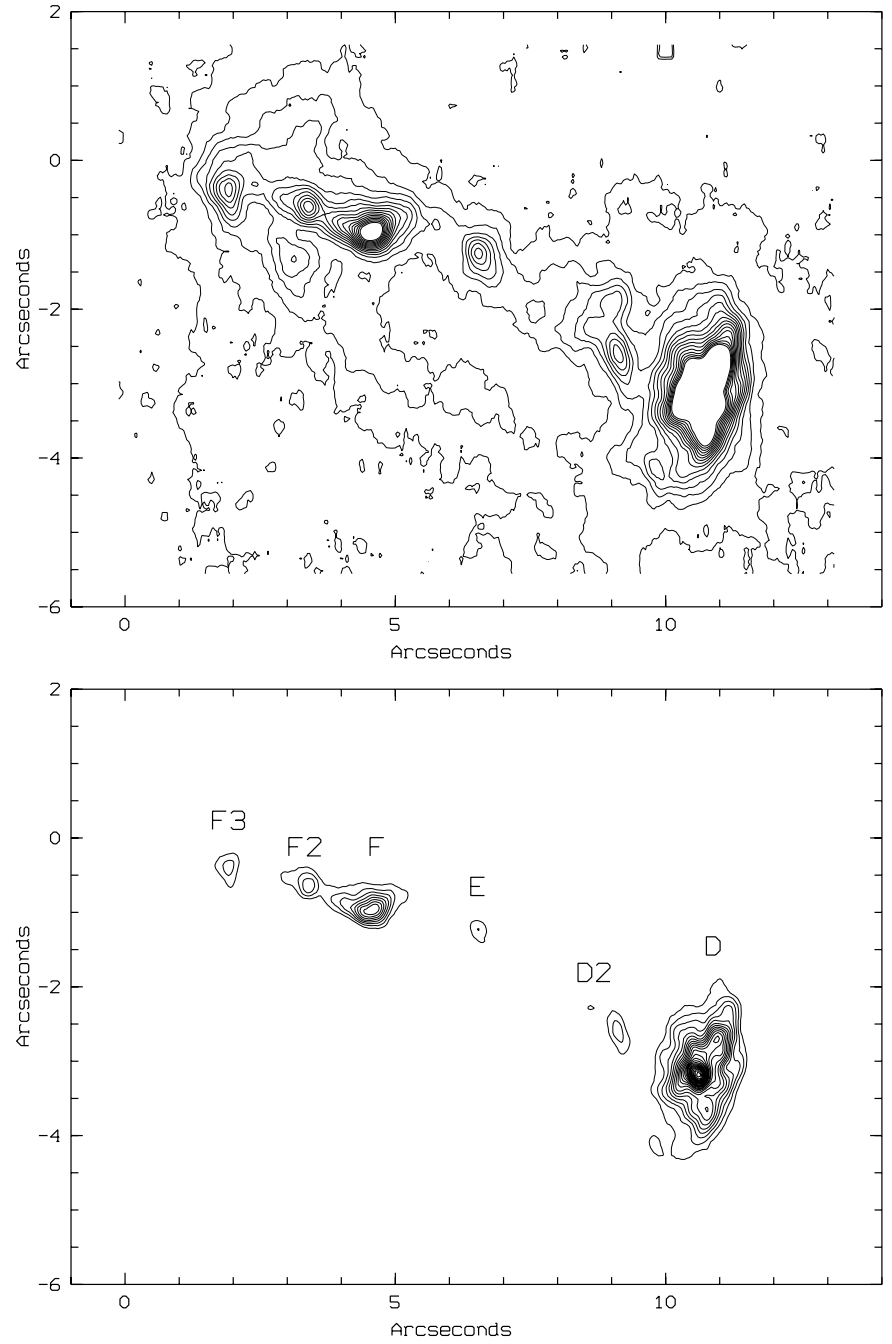

Fig. 1. The 1996 HST $R$-band image displayed as contour plots at two different levels of contrast in order to show both the faint structures and the details in the feature F-D (see text). The $0^{\prime \prime}, 0^{\prime \prime}$ position is the nominal L1551 IRS5 position. In the top panel, the lowest contour is $2.5 \times 10^{-14} \mathrm{erg} \mathrm{cm}^{-2} \mathrm{~s}^{-1}$, and each successive contour is separated by $4.0 \times 10^{-15} \mathrm{erg} \mathrm{cm}^{-2} \mathrm{~s}^{-1}$. In the bottom panel, the values are $5.0 \times$ $10^{-14} \mathrm{erg} \mathrm{cm}^{-2} \mathrm{~s}^{-1}$, and $8.0 \times 10^{-15} \mathrm{erg} \mathrm{cm}^{-2} \mathrm{~s}^{-1}$ respectively.

\section{Results}

\subsection{The wide band HST data}

In Fig. 1, we show the $R$-band representation of the whole jet including the location of the clumps F-D, using designations originally introduced by Neckel \& Staude (1987), and also used and modified by FL94 and FL98. Then, in Fig. 2 we present part of the $I$-band images of the jet. The raw images have a pixel resolution of 0.046 arcsec per pixel. The HST is diffraction limited at the wavelengths in question in this paper, and the PC chip is therefore Nyqvist sampling the PSF. The image scale corresponds at the assumed distance of L1551 to $7 \mathrm{AU}$ or $10^{14} \mathrm{~cm}$ per pixel. The data are presented at two different levels of contrast in order to bring forth both the bright small scale structure and the faint extended emission tracing the outline of the jet. The $R$ - and $I$-band data both show a highly structured jet, but with significant differences. In the $R$-band we can

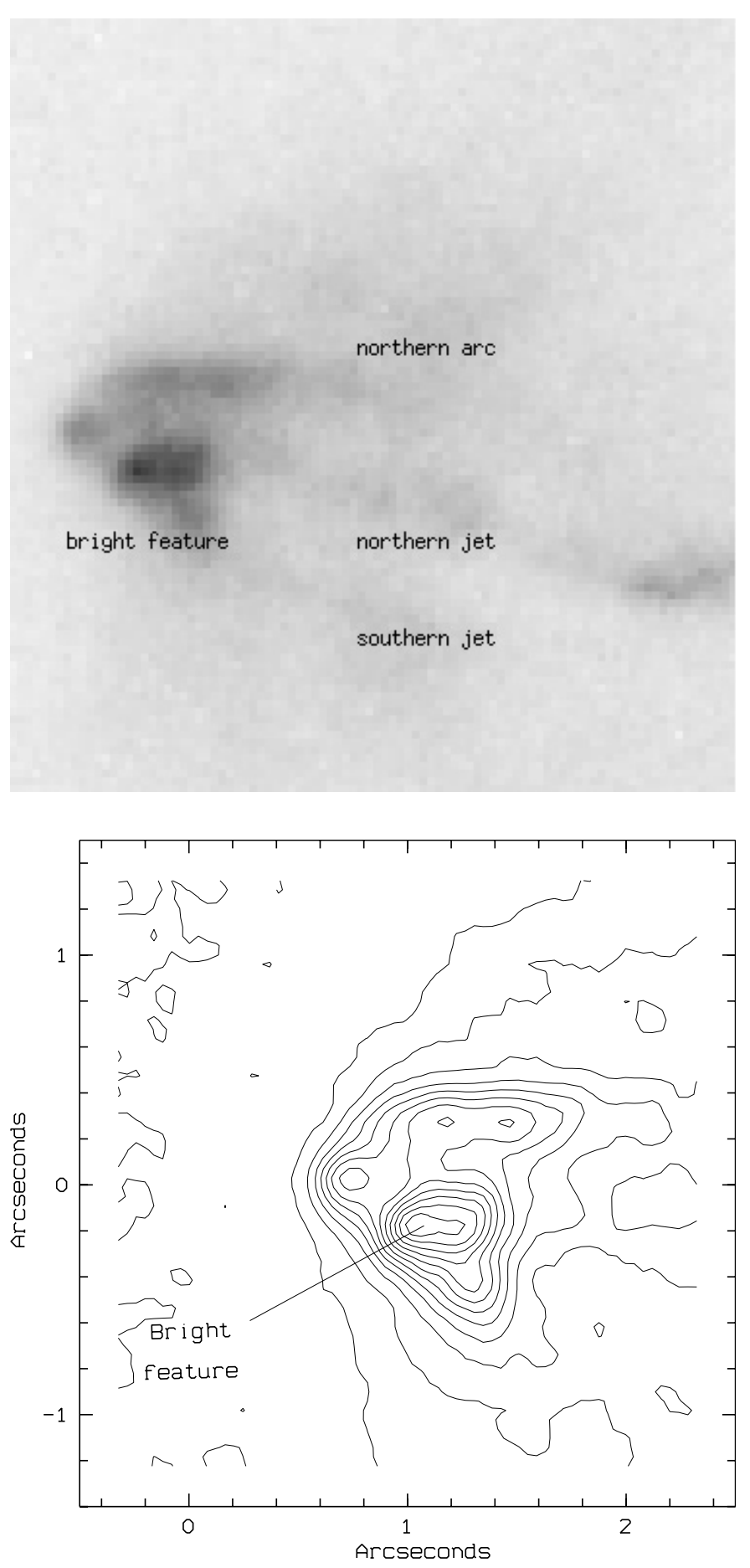

Fig. 2. The inner part of the 1996 HST I-band image displayed as both a contour plot and a grey scale in order to show both faint structures and the details in the "bright feature" (see text). The 0 ", 0 "position is the nominal L1551 IRS5 position. In the bottom panel, the lowest contour value is $5.0 \times 10^{-17} \mathrm{erg} \mathrm{cm}^{-2} \mathrm{~s}^{-1}$, and each contour is separated by $1.4 \times 10^{-17} \mathrm{erg} \mathrm{cm}^{-2} \mathrm{~s}^{-1}$. This data has been smoothed by a Gaussian filter with a 2 pixel radius.

discern several major arc-like components, each consisting of a number of knotlike features. Most obvious, a relatively bright, narrow and "patchy" structure with a clear "wiggle" ends in a very bright feature $\sim 10-12$ arcsec downstream. To conform to FL98 we will designate this the northern jet. The second 
component is fainter, has only 1 or 2 knot-like features before bending back and intersecting the bright feature at the end of the northern jet. Here we designate this feature the southern jet. We also see a faint very narrow and short arc (designated the northern arc), a few tenths of an arcsec north of the bright northern jet (see also Fig. 2).

The $I$-band flux in the inner regions of the jet, are enhanced relative to the flux in the $R$-band image, while the northern jet is relatively weaker in the $I$-band images. All the knots and features visible in the $R$-band can, however, be detected also in the $I$-frame. The $R$-band filter admits emission from the [OI] 6300, $6364 \AA$, [N II] 6548, $6583 \AA$, H $\alpha$ and the [S II] $6717,6731 \AA$ lines, as well as some scattered light.

A few emission lines fall within the band-pass of the $I$-filter viz. [Fe II] $7155 \AA$, [Ca II] $7291 \AA$ and [O II $]+[\mathrm{Ca} \mathrm{II}]$ $7325 \AA$ - see also Cohen \& Fuller (1985). Although, these lines can be seen in our medium and high resolution spectra of the jet to be at significant flux levels $\left(\sim \frac{1}{2} F(\mathrm{H} \alpha)\right.$ for feature D) for a few of the features, as what concerns the innermost part of the jet, they are essentially nonexistent - see Table 4 . The spatial distribution of this emission - as seen in the spectra - is essentially identical to that of the stronger emission lines (e.g. [S II] - see Fig. 8). Features detected in the $I$-band image that have no or little counterpart in the $\mathrm{H} \alpha$ and [S II] images we therefore judge to be primarily due to scattered light.

\subsection{Narrow band HST data}

In Fig. 3 we display contour plots of our $\mathrm{H} \alpha$ and [S II] chosen to both bring out faint details and show changes due to e.g. proper motions.

No trace of the "northern arc", so clearly visible in the I-frame, can be traced in the emission line images. The "southern jet" is visible in the $\mathrm{H} \alpha$ and [S II] images, but it is found that almost all of the line emission originates from the "northern jet". Morphologically, the line emission images look fairly similar to each other. Major differences first become obvious when we analyse the working surface - feature D. In Fig. 5 contour plots showing the detailed structure of the feature D in the light of these two ions, are displayed. The bright core seen in the $R$ - and $I$-band images is obvious in the $\mathrm{H} \alpha$ image, but less so in the [S II] data. Instead in the latter band we see a mottled structure with a number of point-like emission centers with the brightest emission further away from IRS5 than the bright core seen in the $\mathrm{H} \alpha$ data (see also Fig. 6). Referring to the ground based data of FL94 it was also remarked upon that the [S II] intensity maxima were "further out" from IRS5.

Using images obtained under sub-arcsec seeing conditions, and then applying an unsharp mask, FL94 found a separation of $\mathrm{H} \alpha$ and [S II] of 0.5 arcsec. This is confirmed by the present HST data set.

\subsection{Spectroscopic results}

In Tables 3 and 4 we present a summary of our spectroscopic results. The high spectral resolution data obtained towards the working surface (feature D), is presented in the first table. Here the peak radial velocity is found between $-160 \mathrm{~km} \mathrm{~s}^{-1}$ and $-185 \mathrm{~km} \mathrm{~s}^{-1}$. The maximum FWZI (Full Width at Zero Intensity) is $\sim 300 \mathrm{~km} \mathrm{~s}^{-1}$, which should be identified with the shock velocity at the apex (Hartigan et al. 1987). This is also confirmed by the observation of [O III] emission from the working surface by Fridlund et al. (2005).

In Table 4, we show the medium resolution spectroscopy obtained through slits positioned such as to map out the radial velocity field of the two jets. Also in Fig. 8 we demonstrate both the positions where the spectraq were obtained, as well as samples of the spectroscopic information ( $\mathrm{H} \alpha$ and [Fe II] $7155 \AA$ ). These spectra clearly show our conclusions drawn about the velocity field in Sects. 3.4, 4.1 and 4.2. From the spectroscopic data, it is clear, that there is indeed two velocity systems connected with the jet. A comparison between the spectroscopy and the images allow a one-to-one correspondence between the identified features (F3-D), and the velocity components in the spectra. These results are presented in detail in Table 4. The highest radial velocities are found to be associated with the northern, brighter component, where essentially all of the visible shock induced knots are found. A lower (more positive) radial velocity system is connected with the southern component. The highest velocity found in any ion within the jet appear to be associated with the innermost features $(\mathrm{F}$, F2), where peak velocities of $430 \mathrm{~km} \mathrm{~s}^{-1}$, and $F W Z I$ of up to $400 \mathrm{~km} \mathrm{~s}^{-1}$ are present (see also Sect. 4.2). A comparison between our data and the IR [Fe II] spectroscopy of Pyo et al. (2002) is fully consistent when taking into account differences in sensitivity. A more thorough discussion about the spectroscopic results can be found in Liseau et al. (2005) and Fridlund et al. (2005).

\subsection{The nature and proper motions of the clumps along the jets}

With one major exception, all well defined features that can be identified in both HST epochs are to be found in the northern jet. The exception is the bright oval feature seen in the $I$-band (Fig. 2, see also discussion).

Proper motions of features in the jet were found by Neckel \& Staude (1987), and further studied by FL94. These ground based observations led to the identification of the features denoted A-F. Features A and B are found further out than the others and are thus not on the PC chip of the WFPC2. We refer to FL94 for illustration. In both Neckel \& Staude (1987) and FL94 it was found that distinct new knots appeared from behind the obscuration along the line of sight to IRS5. FL94 determined the transverse velocities for knots $\mathrm{D}, \mathrm{E}$ and $\mathrm{F}$ to be between $120 \mathrm{~km} \mathrm{~s}^{-1}$ and $\sim 300 \mathrm{~km} \mathrm{~s}^{-1}$. Knot E appeared well defined in the middle of the jet in the data from 1989, but in 1993 it seemed on the verge of merging with the working surface D. At the same time there were indications of a new feature appearing at the original spot of E. When comparing the separate [S II] and $\mathrm{H} \alpha$ images, we find that the features appear to be well defined in each filterband image, but are found at somewhat different positions. This could be explained essentially through a change of excitation conditions at different positions (see Table 5 and Figs. 3 and 4). In some of the features there 

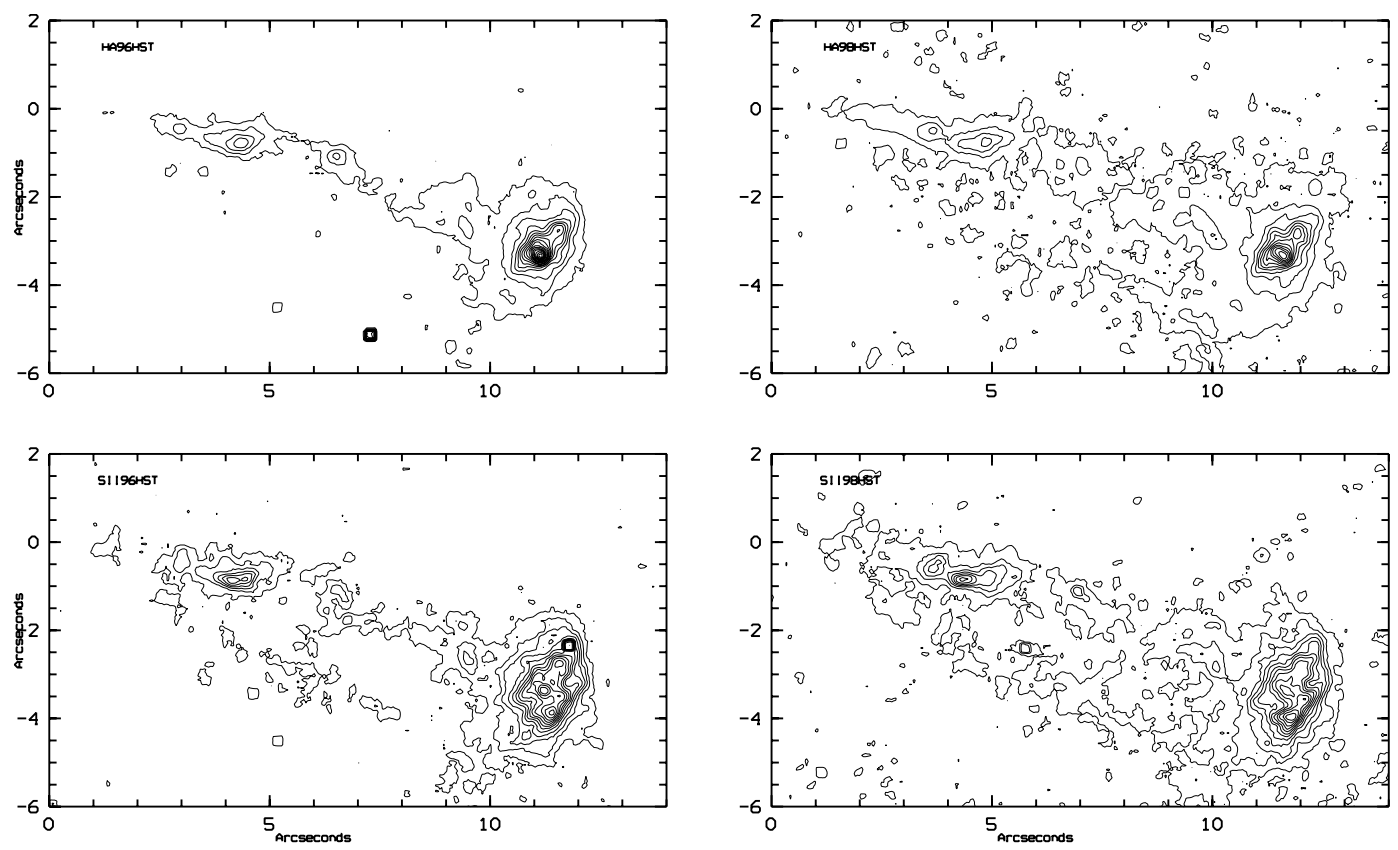

Fig. 3. The 1996 (left upper panel) and 1998 (right upper panel) $\mathrm{H} \alpha$ images displayed as contour plots. The lowest contour is at the $3 \sigma$ level, and each consequtive contour is at intervals of $1 \sigma$. The $0^{\prime \prime}, 0^{\prime \prime}$ position is the same as in Figs. 1 and 2 . Note that the innermost part of the jet (position F3 in the $R$-band image) is not seen in emission. The bright spike in the top panel (7".3, -4 .' 8 is a Cosmic ray hit.) The 1996 [S II] image (bottom left panel) and 1998 [S II] (bottom right panel) imagesare also displayed as contour plots with the same contours. The bright pointlike knot in the lower left panel at position $11^{\prime \prime} .5,-2{ }^{\prime \prime} 5$ is a cosmic ray strike that could not be removed. All images are obtained with the same exposure time.

are indications of them being representative of clumps of gas moving at a high speed through the surrounding gas (so called interstellar bullet, e.g. Norman \& Silk 1979). This is especially true for knot E, which is compact and appears at different positions at the two HST epochs. This feature is, however, currently traveling at transverse velocities about half of those measured by FL94. This behavior could be explained by the present feature $\mathrm{E}$ being either:

- a different bullet - ejected at a different velocity;

- the feature E observed by FL94 - from the ground - was the seeing-smoothed agglomeration of several "bullets";

- a combination of both these options.

As is evident from Table 5, the knots D2 and F2 (see Fig. 1) are the knots with the highest velocities, consistent with the results of FL94.

Our radial velocity results (see Fig. 8) indicate that the highest velocities for a given inclination should be found as far in towards IRS5 as possible, which is consistent with F2 having the highest transverse velocity (see Table 5). In Fig. 4, we have zoomed in on the area around knot F. A number of smaller, well defined (sub-)features are obvious in the images. This F-complex consist of sub-features F2, $\mathrm{F}^{\prime}$ and F. The F2 knot is identifiable as a bright isolated knot, of intermediate excitation, seen in both filters and in both epochs, while $\mathrm{F}^{\prime}$ has low-excitation characteristics and is harder to identify unambiguously in all filters/epochs. Finally, the F feature is of higher excitation.

The lengthening of the jet with time, as measured by its total length at each epoch is shown in Table 6, where we have collected all the data for this parameter that we have available. The average change in length found during this period (1 Dec. 1989 to 21 Oct. $2001=4342$ days) is 0.3 arcsec $\mathrm{yr}^{-1}$ representative of a transversal velocity of $235 \mathrm{~km} \mathrm{~s}^{-1}$.

In Table 7, we compare the velocity in the plane of the sky of the knots A, B, and C (see FL94 for location) over total timespan covered by the data with the results in our earlier paper. There is maybe an indication that the knots are slowing down as they progress further out beyond the working surface, as well as becoming somewhat less well defined. This needs to be confirmed with observations over a longer timebase. Nevertheless, these features have now existed as well defined entities since at least 1983 (Neckel \& Staude 1987). In the meantime they have moved between 600 and $1000 \mathrm{AU}$ further downstream. The northern jet appears to be wiggly - particularly in the $\mathrm{H} \alpha$ band image. The amplitude of the excursions is $\sim 0.5$ arcsec, and the distance between the nodes is 3 arcsec or $\sim 450$ AU $\left(\sim 7 \times 10^{15} \mathrm{~cm}\right)$. In unpublished ground based data from $1996(\mathrm{H} \alpha)$, we have used an unsharp mask on the data, and thanks to a much higher signal-to-noise ratio confirmed the size and extent of the pattern.

FL98 interpreted the HST data from 1996 as indicative of at least two physically separated velocity components of the jet. In our new spectroscopic observations we have complete coverage of the jet with medium and high resolution spectroscopy, which allows us to determine the velocity field of the entire jet (Fig. 8). From different line ratios we can determine some of the physical parameters. In order to do so, we use previously unpublished data, where we have imaged the whole SW L1551 complex through narrow interference filters. 

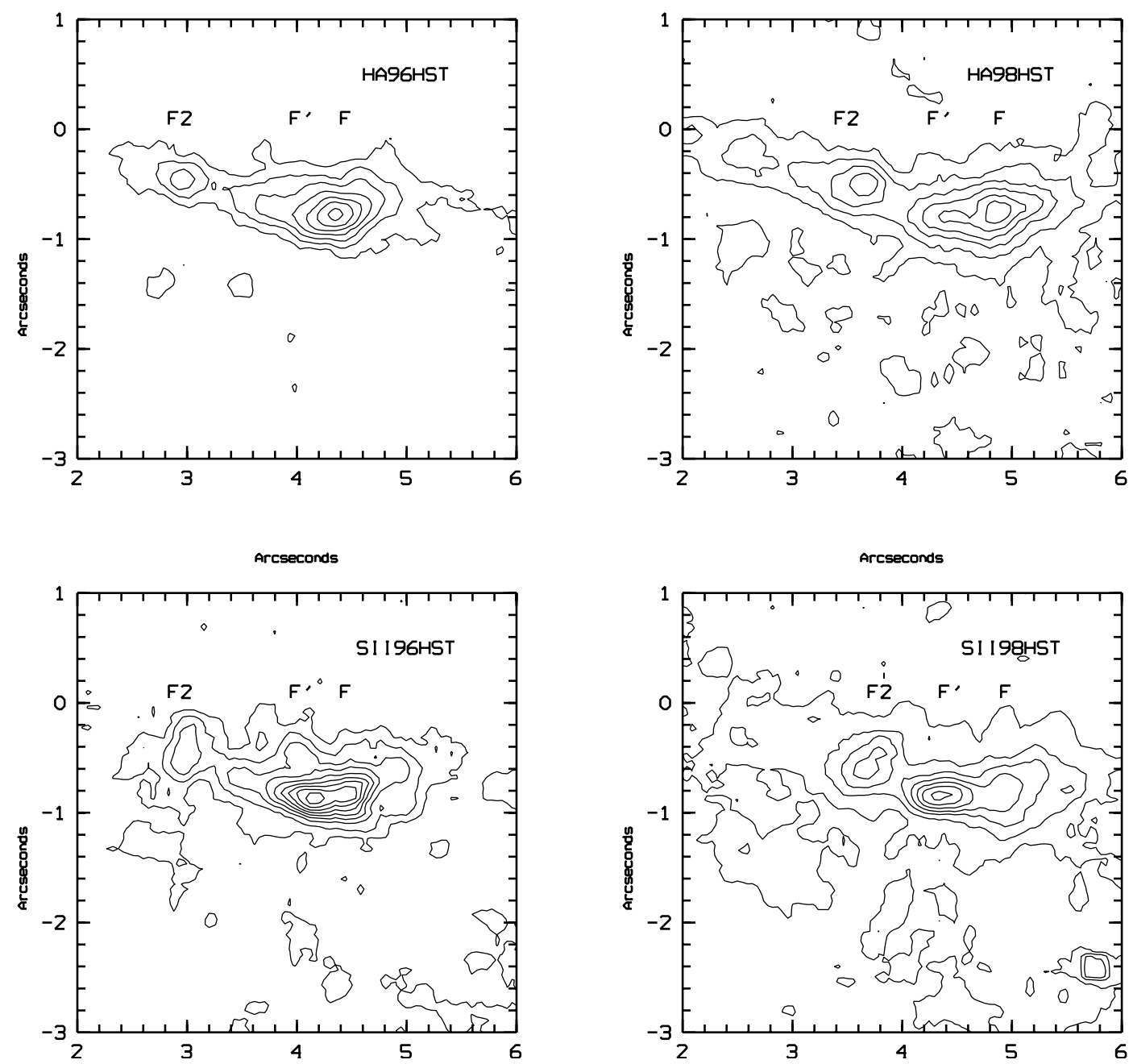

Fig. 4. The 1996 (left) and 1998 (right) $\mathrm{H} \alpha$ (Top) and [S II] (Bottom) contour representations of the region around knot F. North is up and East towards the left. The lowest contour is $2 \times 10^{-18} \mathrm{erg} \mathrm{cm}^{-2} \mathrm{~s}^{-1}$ pixel $^{-1},(3 \sigma)$ and each consecutive contour is separated by $1 \sigma$. Note the proper motions over the 770 days between the 2 epochs representative of velocities of between $120 \mathrm{~km} \mathrm{~s}^{-1}$ and $250 \mathrm{~km} \mathrm{~s}^{-1}$ if representative of actual motions.

Those filters included $\mathrm{H} \alpha, \mathrm{H} \beta$ and the [S II] $4067 / 68 \AA$ ion. We can then a) deredden our data and b) determine the electron temperature and electron density along the jet, and in and around the working surface (knot D). The electron density, $n_{\mathrm{e}}$, can be determined from the [S II] $6717 \AA /[$ S II] $6731 \AA$ ratio, and a suitable model. We use the same recent 5-level model for [S II] as Fridlund et al. (1998). The electron temperature, $T_{\mathrm{e}}$, can be determined from the same data together with information about the flux from the [S II] 4067/68 A. Although inherently faint, both knot $\mathrm{F}$ and knot $\mathrm{D}$ are detected in the blue [S II] lines, and with fluxes of $1.25 \times 10^{-16} \mathrm{erg} \mathrm{cm}^{-2} \mathrm{~s}^{-1}$ $(S / N \sim 4)$ and $7.05 \times 10^{-16} \mathrm{erg} \mathrm{cm}^{-2} \mathrm{~s}^{-1}(S / N \sim 11)$. Note that the apertures used are different. Dereddening is achieved from the $\mathrm{H} \alpha / \mathrm{H} \beta$ ratio (see Tables 1 and 2). Using the above mentioned spectroscopic data, and our [S II] model, we arrive at values of $T_{\mathrm{e}}(\mathrm{D}) \sim 8700 \mathrm{~K} \pm 1000 \mathrm{~K}$, $T_{\mathrm{e}}(\mathrm{F}) \sim 14250 \mathrm{~K} \pm 1750 \mathrm{~K}, n_{\mathrm{e}}(\mathrm{D}) \sim 1600 \mathrm{~cm}^{-3} \pm 200 \mathrm{~cm}^{-3}$, and $n_{\mathrm{e}}(\mathrm{F}) \sim 1800 \mathrm{~cm}^{-3} \pm 200 \mathrm{~cm}^{-3}$. Note that these values then are averages over the aperture used in each case. For knot $\mathrm{F}$ this is about $300 \times 300 \mathrm{AU}^{2}$, while for feature $\mathrm{D}$ it is $\sim 530 \times 530 \mathrm{AU}^{2}$, assuming a distance of $\sim 150 \mathrm{pc}$.
We have also analysed our [Fe II] data using a model atom including 142 levels with 1438 transitions. Our model incorporates radiative transfer, but in this case this is unnecessary since the optical depth in the [Fe II] lines is of order $10^{-8}$. We have detections of [Fe II] 7155.1, $7172.0 \AA$, $7452.5 \AA$ and $8619.3 \AA$ for knot $\mathrm{D}$, and our best fit indicates higher $T_{\mathrm{e}}$ and $n_{\mathrm{e}}$ than the [S II] data, viz. $13500 \mathrm{~K}$ and $3200 \mathrm{~cm}^{-3}$, respectively. Given the somewhat different apertures through which we are averaging our data, the values are quite reasonable, however. A puzzling result, however, is the absence of the [Fe II] line at $6875.8 \AA$. In our model, which assumes a solar abundance, the line at $7454.6 \AA$ should always be weaker. We note, however, that in observations of $\mathrm{HH} 1$, the same situation occurs, i.e. the detection of $7454.6 \AA, 8619.3 \AA$, but not $6875.8 \AA$ (Solf et al. 1988).This suggests an explanation. Like in the case of HH1, we do not detect any permitted FeII lines. As pointed out by Solf et al. (1988), the permitted FeII lines require an excitation energy of, on average, $5.2 \mathrm{eV}$, while the forbidden lines have lower excitation energy. All of the detected lines in our spectrum have excitation energies of $1.7-2.0 \mathrm{eV}$, while the $6875.8 \AA$ line, falls in between since it requires 

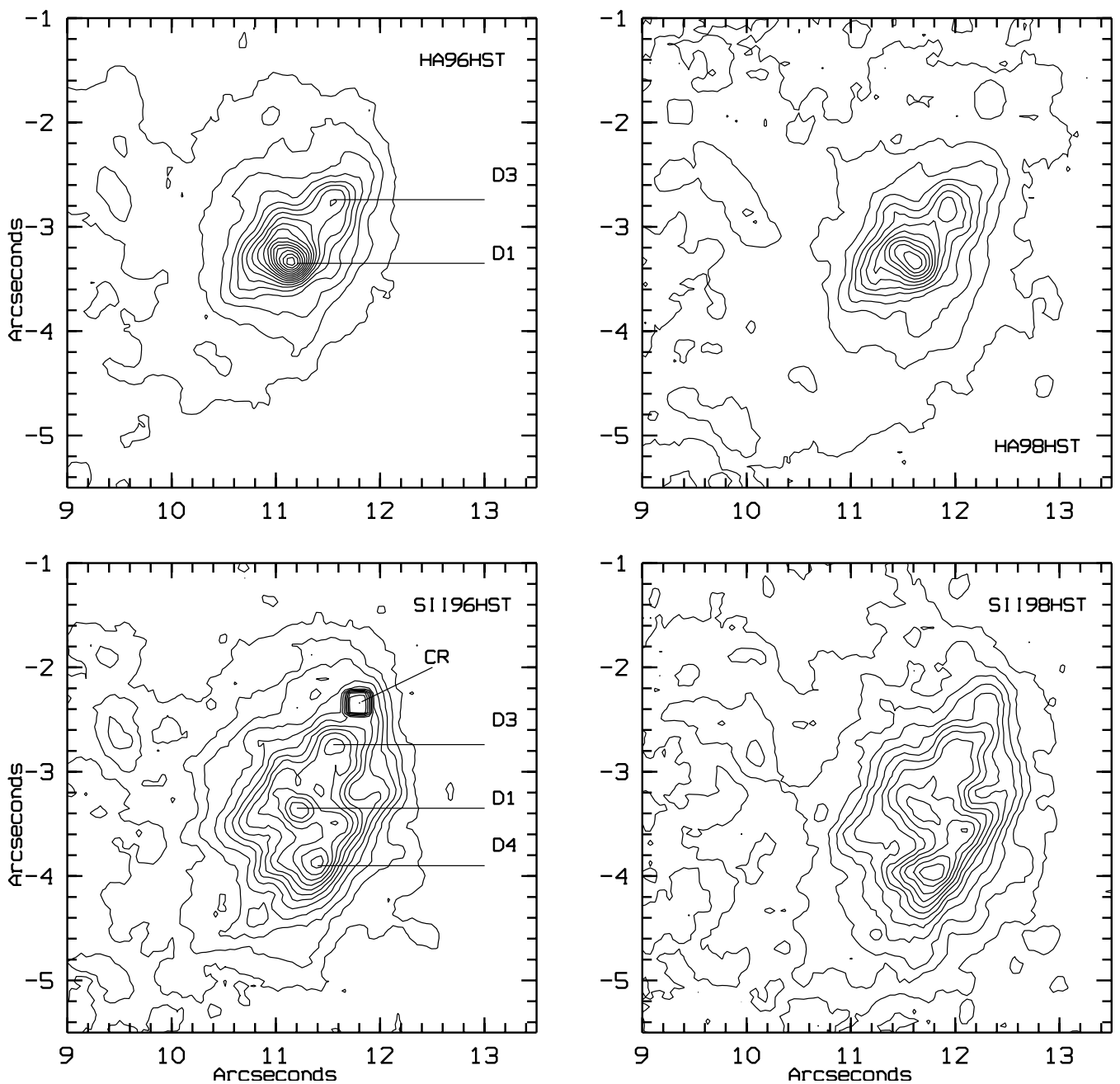

Fig. 5. The 1996 and $1998 \mathrm{H} \alpha$ (top) and [S II] (bottom) contour representations of feature D - the presumed working surface. North is up and East towards the left. The lowest contour is $2.5 \times 10^{-18} \mathrm{erg} \mathrm{cm}^{-2} \mathrm{~s}^{-1}$ pixel $^{-1}$, and each contour is separated by $1.0 \times 10^{-18} \mathrm{erg} \mathrm{cm}^{-2} \mathrm{~s}^{-1} \mathrm{pixel}^{-1}$. The bright, compact feature, dominating the $\mathrm{H} \alpha$ frame has been given the designation D1 in this paper. Note the proper motions over the 770 days between the 2 epochs representative of transverse velocities of about $150 \mathrm{~km} \mathrm{~s}^{-1}$. CR is a cosmic ray strike.

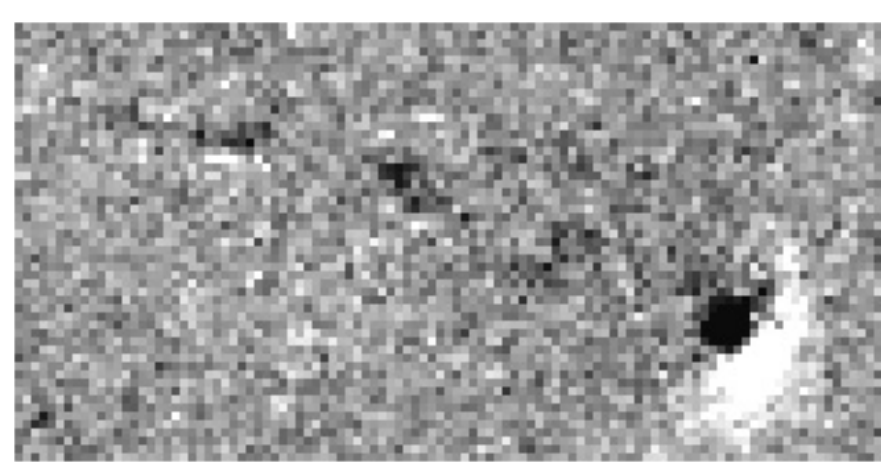

Fig. 6. An $\mathrm{H} \alpha$-[S II] $6717+6731 \AA ̊$ difference image (1996 data) of the complete jet, shown as a greyscale. Here $\mathrm{H} \alpha$ is represented by black, while the [S II] is white. The picture is smoothed to 0.1 arcsec resolution, and the image is 11 arcsec by 6 arcsec. The strong white/black feature is knot $\mathrm{D}$ - the working surface. The spatial separation of black $(\mathrm{H} \alpha)$ and white([S II] ) is interpreted in the text as the resolving of the shock into the Mach disk (black) and forward- or bow-shock (white). Note that apart from the working surface the rest of the jet cancels out almost perfectly, leaving only a few filaments of separated emission. $\sim 3.8 \mathrm{eV}$. It appears thus that the kinetic energies in knot D are not high enough to excite forbidden lines with higher energies than $\sim 2.0 \mathrm{eV}$. There is no indication of any line with a higher excitation energy than that, although a marginal detection of $6875.8 \AA$ is found at the location of knot F, and at a radial velocity of $\sim-300 \mathrm{~km} \mathrm{~s}^{-1}$. An alternative explanation is that the atomic data, as given in Quinet et al. (1996), for the $6875.81 \AA$ line are in error.

\subsection{The extinction towards the jet}

In Table 1 we present the flux from some of the persistent features found along the jet as a function of time (including some of our unpublished data). The presence of $\mathrm{H} \beta$ emission allows us to determine the extinction towards separate parts of the jet (assuming the appropriate recombination case), where one would expect the extinction to grow as one progresses towards the position of IRS5.

Fridlund et al. (2005) have detected [O III] emission that can be taken as direct evidence of a strong shock being present 
Table 1. Observed fluxes ( $\mathrm{erg} \mathrm{cm}^{-2} \mathrm{~s}^{-1}$ ) for the assumed working surface (knot D), the feature $\mathrm{F}$, and the total jet in in all of the imaging data. Note that these data have not been de-reddened. The quoted accuracy includes both the estimated precision in the flux calibration and the measurement error.

\begin{tabular}{llllll}
\hline \hline Feature & $\mathrm{H} \alpha$ & $\mathrm{H} \beta$ & {$[\mathrm{S} \mathrm{II}]$} & Source & $\begin{array}{l}\text { Accu- } \\
\text { racy }(\%)\end{array}$ \\
\hline $\mathrm{D}$ & $1.8 \times 10^{-14}$ & - & $1.8 \times 10^{-14}$ & FL94 & 10 \\
$\mathrm{~F}$ & $5.2 \times 10^{-15}$ & - & $3.0 \times 10^{-15}$ & FL94 & 10 \\
$\Sigma_{\text {Alljet }}$ & $3.3 \times 10^{-14}$ & - & $2.9 \times 10^{-14}$ & FL94 & 10 \\
$\mathrm{D}$ & $2.1 \times 10^{-14}$ & - & $2.2 \times 10^{-14}$ & HST 1996 & 5 \\
$\mathrm{~F}$ & $3.8 \times 10^{-15}$ & - & $3.4 \times 10^{-15}$ & HST 1996 & 5 \\
$\Sigma_{\text {Alljet }}$ & $5.2 \times 10^{-14}$ & - & $4.8 \times 10^{-14}$ & HST 1996 & 5 \\
$\mathrm{D}$ & $1.8 \times 10^{-14}$ & $2.3 \times 10^{-15}$ & - & NOT 1996 & 10 \\
$\mathrm{~F}$ & $2.7 \times 10^{-15}$ & $2.3 \times 10^{-16}$ & - & NOT 1996 & 10 \\
$\Sigma_{\text {Alljet }}$ & $4.1 \times 10^{-14}$ & $5.0 \times 10^{-15}$ & - & NOT 1996 & 10 \\
$\mathrm{D}$ & $1.8 \times 10^{-14}$ & - & $2.0 \times 10^{-14}$ & HST 1998 & 5 \\
$\mathrm{~F}$ & $3.4 \times 10^{-15}$ & - & $3.3 \times 10^{-15}$ & HST 1998 & 5 \\
$\Sigma_{\text {Alljet }}$ & $4.3 \times 10^{-14}$ & - & $4.2 \times 10^{-14}$ & HST 1998 & 5 \\
$\mathrm{D}$ & $2.0 \times 10^{-14}$ & - & $1.7 \times 10^{-14}$ & NTT 1998 & 10 \\
$\mathrm{D}$ & $1.8 \times 10^{-14}$ & $2.4 \times 10^{-15}$ & - & NOT 2001 & 10 \\
$\mathrm{~F}$ & $3.8 \times 10^{-15}$ & $3.2 \times 10^{-16}$ & - & NOT 2001 & 10 \\
$\Sigma_{\text {Alljet }}$ & $4.1 \times 10^{-14}$ & $5.2 \times 10^{-15}$ & - & NOT 2001 & 10 \\
\hline
\end{tabular}

Table 2. Extinction determined from the $\mathrm{H} \alpha / \mathrm{H} \beta$ ratio, at two epochs (December 1996 and October 2001) for several features in the jet (see text). The accuracy in the determination of the ratios is $\sim 20 \%$. Data taken from Fridlund et al. (2005).

\begin{tabular}{|c|c|c|c|c|}
\hline Feature & Ratio $_{1996}$ & $\overline{\text { Ratio }_{2001}}$ & $\begin{array}{l}A_{\mathrm{V}}(1996) \\
R=3\end{array}$ & $\begin{array}{l}A_{\mathrm{V}}(2001) \\
R=3\end{array}$ \\
\hline$\overline{\mathrm{D}}$ & 8.5 & 7.0 & 2.8 & 2.2 \\
\hline E & 8.0 & 10. & 2.6 & 3.2 \\
\hline $\mathrm{F}$ & 11.9 & 11.9 & 3.7 & 3.7 \\
\hline $\begin{array}{l}\text { Region between } \\
\mathrm{F} \text { and end } \\
\text { of jet }(\mathrm{F} 2)\end{array}$ & $12-15$ & $14-20$ & $4-4.5$ & $4.2-5.1$ \\
\hline
\end{tabular}

at the position of knot D. Tentatively, we then identify the feature D as a (relatively) strong shock. Somewhat weaker [O III] emission is also detected from the position of knot F. It is at these positions we then expect that the collisional contribution to the population of $\mathrm{H} \alpha$ to be insignificant (Hartigan et al. 1987). We can thus use the Balmer decrement for case B recombination in order to estimate the extinction.

As can be seen from Tables 1 and 2, in 1996 we find a ratio of $\mathrm{H} \alpha / \mathrm{H} \beta$ of 8.5 for feature $\mathrm{D}$, while the data obtained in 2001 indicate a ratio of 7.0 for the same object. During the intervening time, this feature has moved away from IRS5 at a rate of $\sim 0.2 \operatorname{arcsec}_{\mathrm{yr}^{-1}}$ (Table 5). Cohen \& Fuller (1985) found a $\mathrm{H} \alpha$ to $\mathrm{H} \beta$ ratio of $\sim 25$. This value, however, should be used with some caution, since we note that they observed using blind offsets from a nearby star, and the $\mathrm{H} \alpha$ and the $\mathrm{H} \beta$ spectra were obtained at different times (pointings). There could thus be an offset by an unknown amount between $\mathrm{H} \alpha$ and $\mathrm{H} \beta$. Further, our data indicate that the proper motion of this feature over $\sim 13$ years, would implicate a displacement of $\sim 2-3$ arc$\mathrm{sec}$, which means that the physical conditions could be very different.
Using a normal galactic extinction curve (Rieke \& Lebofsky 1985) our data for knot $\mathrm{D}$ indicate $A_{\mathrm{V}} \sim 3$ mag in 1996 and $\sim 2 \mathrm{mag}$ in 2001. A $\theta$ Orionis extinction law (Cardelli \& Clayton 1988) gives values of 3.6 and 2.9 mag of visual extinction respectively. Applying the data of Cohen and Fuller (1985 - data obtained 1982) we have a visual extinction of 5.8 and 7.4 mag depending on the extinction law applied.

As we progress further in along the jet, towards IRS5, the extinction increases. It is close to $4 \mathrm{mag}$ at the position of feature F. Towards the very tip of the jets, i.e. the apex where they suddenly emerge from invisibility, we find that the ratio is indicative of an extinction of 4 to 6 mag. These values are consistent with the extinction calculated from the X-ray spectrum for the L1551 IRS5 source (Favata et al. 2002).

\section{Discussion}

\subsection{The working surface and the physical conditions along the jets}

The first, most easily accessed, physical parameter determined from our HST data is the level of excitation as defined by the ratio of [S II] flux to H $\alpha$ flux (Raga et al. 1996). For the purpose of this paper we will call this ratio "the excitation index". To display the positions where the low excitation [S II] emission dominates over the $\mathrm{H} \alpha$ emission - and vice versa we have computed the difference images between $\mathrm{H} \alpha$ flux and [S II] flux (e.g. Fig. 6). In this figure the most obvious feature is the end of the jet (Feature D) where prominent [S II] , representative of low-excitation emission and displayed in white is wrapping around the prominent $\mathrm{H} \alpha$ (displayed in black) which is representative of high-excitation emission. Since we know the direction and rough inclination of the jet, the morphology leads to the interpretation as being a nice example of a working surface with the bow-shock (strong [S II] ) wrapping itself around the Mach-disk (strong $\mathrm{H} \alpha$ ). 
Table 3. Ions detected in spectroscopic observations with the ESO NTT-EMMI instrument. These observations only covered knot D (brightest feature in Fig. 1). Note that the data are not de-reddened. Note also that the values in the table refer to the maximum values. There is a large variation between slit positions for different lines. These differences presumably are due to a geometric effect (working surface spatially resolved), discussed in the text. The accuracy in the flux determination is approximately $10 \%$, and this error includes both the error in the calibration and the actual measurement error. The error in velocity is $10 \mathrm{~km} \mathrm{~s}^{-1}$.

\begin{tabular}{llllll}
\hline \hline Ion & $\begin{array}{l}\text { Rest wavelength } \\
(\AA)\end{array}$ & $\begin{array}{l}\text { Max line width } \\
\left(F W Z I \mathrm{~km} \mathrm{~s}^{-1}\right)\end{array}$ & $\begin{array}{l}\text { Max peak velocity } \\
\left(\mathrm{km} \mathrm{s}^{-1}\right)\end{array}$ & $\begin{array}{l}\text { Total flux observed } \\
\left(10^{-15} \mathrm{erg} \mathrm{cm}^{-2} \mathrm{~s}^{-1}\right)\end{array}$ & Comment \\
\hline$[\mathrm{OI}]$ & 6300.2 & 180 & -170 & 5.2 & \\
{$[\mathrm{OI}]$} & 6363.8 & 185 & -175 & 5.3 & \\
{$[\mathrm{NII}]$} & 6548.1 & 65 & -185 & 0.63 & \\
$\mathrm{H} \alpha$ & 6562.8 & 310 & -175 & 20.0 & several separate components ${ }^{1}$ \\
{$[\mathrm{NII}]$} & 6583.6 & 270 & -185 & 7.0 & $\Sigma_{\text {flux }(6717+6731)}=17.2 \times 10^{-15} \mathrm{erg} \mathrm{cm}^{-2} \mathrm{~s}^{-1}$ \\
{$[\mathrm{SII}]$} & 6716.4 & 200 & -175 & 7.26 & \\
{$[\mathrm{SII}]$} & 6730.8 & 220 & -170 & 9.9 & several separate components ${ }^{1}$ \\
{$[\mathrm{FeII}]$} & 7155.1 & 250 & -170 & 2.6 & \\
{$[\mathrm{CaII}]$} & 7291.5 & 240 & -180 & 6.5 & Detected at $3 \sigma-$ level \\
{$[\mathrm{OII}]$} & 7319.4 & - & - & - & 3.0 \\
{$[\mathrm{CaII}]$} & 7323.9 & 125 & -160 & 0.16 & \\
{$[\mathrm{OII}]$} & 7329.9 & - & - & &
\end{tabular}

${ }^{1}$ Values in table refer to the strongest component.

Taken together with the excitation index, as defined above, the ratio of $[\mathrm{O} \mathrm{III}] / \mathrm{H} \beta$ refines the understanding of the excitation conditions. According to Raga et al. (1996), a value of [O III] / $\mathrm{H} \beta$ larger than 0.1 together with a [S II] $/ \mathrm{H} \alpha$ smaller than 1.5 indicates high excitation, while opposite values are indicative of very low excitation conditions. Fridlund et al. (2005) have found [O III] $5007 \AA$ emission emanating from both features $\mathrm{F}$ and $\mathrm{D}$, as well as $\mathrm{H} \beta$ emission distributed in the same way as the $\mathrm{H} \alpha$ emission (see discussion about the extinction above).

Feature $\mathrm{F}$ is found by Fridlund et al. (2005) to have an [O III] $/ \mathrm{H} \beta$ ratio of $0.3 \pm 0.06$, while the ratio for knot $\mathrm{D} 1$ is $0.22 \pm 0.02$, both ratios measured in 2001 . These are values indicative of a high level of excitation. For the [S II] $/ \mathrm{H} \alpha$ ratio we have data covering a larger time span. Feature F (see Fig. 1) has an [S II] $/ \mathrm{H} \alpha$ ratio varying from $0.6 \pm 0.1$ in 1994 to $1 \pm 0.1$ in 1998 , thus indicating that an initial high excitation feature is somewhat weakening with time. An analysis of the F-complex in the HST data shows that the 3 features F2, F and $\mathrm{F}^{\prime}$ had $[\mathrm{S} \mathrm{II}] / \mathrm{H} \alpha$ ratios of $1.0 \pm 0.1,1.9 \pm 0.2$ and $0.9 \pm 0.1$ in 1996. In 1998 the values for the ratios were $1 \pm 0.1,0.9 \pm 0.1$ and $0.8 \pm 0.1$ respectively. The feature $\mathrm{F}$ as observed from the ground, is of course the agglomeration of all three subcomponents. These observations demonstrate the occurrence of changes in the jet not only with respect to the morphological properties, but also to the physical conditions within a time scale of a few years. Knot $\mathrm{F}$ is also the second brightest emission feature along the two jets. Careful analysis of the HST data show that a very high level of excitation is mainly found along the outer (northern) edge of feature F, while a very thin ( $\sim$ a few pixel thick) layer of lower excitation (demonstrated by strong [S II] emission in our difference images) is found along the southern edge of the jet. Taken together with the $[\mathrm{O}$ III] $/ \mathrm{H} \beta$ index mentioned above we conclude that (at least parts of) knot $\mathrm{F}$ is of high but variable excitation in the classification scheme of Raga et al. (1996).
The fainter feature $\mathrm{E}$ has an $[\mathrm{S} \mathrm{II}] / \mathrm{H} \alpha$ ratio of 0.6 both in 1996 and in the 1998 data. This is thus indicative of a relatively high level of excitation, regardless of the fact that the feature has moved a significant distance in the intervening time. It is, however, not detected in [O III] by Fridlund et al. (2005).

Finally, D, the working surface, is found to have an average level of excitation index of $\sim 1$, with very little variation over time (see Table 1). If we analyse the separate, very bright knots that make up what we have been calling feature D, we find a more complex picture. In the $\mathrm{H} \alpha$ and the [S II] images (see Fig. 5), we see a number of bright (sub-)features. The brightest of these are the ones where we argued in FL98 that they were the signature of the working surface of the jet with the Mach disk brightest in $\mathrm{H} \alpha$ and the forward shock (or bow-shock) being brightest in [S II] respectively. They are now designated D1 (brightest in $\mathrm{H} \alpha$ ) and D4 (brightest in SII). D1 has an excitation index of $\sim 0.5$ making it a high excitation object. This is also supported by the $[\mathrm{O} \mathrm{III}] / \mathrm{H} \beta$ ratio of 0.22 (see above). The opposite is true for D4 as well as for the "bow-shape" surrounding the core. Here we find excitation indeces of $\sim 1-5$ indicating low-excitation conditions.

We already identified the entire feature D as the working surface of the jet in FL98. The characteristic features allowing us to make this identification are clearly visible in Fig. 6. Also the interface between the regions dominated by $\mathrm{H} \alpha$ and [S II] emission is clearly discernible in Fig. 7 which shows contour plots of the two HST epochs and where the [S II] emission has been subtracted from the $\mathrm{H} \alpha$ emission. The latter emission is strongly concentrated towards the inner part of the working surface, at the position of the high excitation core, while the [S II] is "swept" into a nice bow-shape surrounding it. The steepest gradient of the $\mathrm{H} \alpha$ emission is on the side away from IRS5 and on the interface with the region dominated by [S II]. Comparing with the individual images of $\mathrm{H} \alpha$ and [S II] in Fig. 5 we see that the [S II] have its steepest gradient where the bow-shape is furthest away from IRS5. In the context of the model of Hartigan (1989), we interpret these gradients to represent the locations 
Table 4. Ions detected in medium resolution spectroscopic observations with the Nordic Optical Telescope ALFOSC instrument. These observations covered essentially the whole jet (one slit position missing, see Fig. 8). Note also that the values in the table refer to the maximum values. There is a large variation between different slit positions for the same ions. These differences presumably are caused by the two distinct jets. The total flux refers to the flux from all of the jet (excluding that emanating from the missing slit position). Note that the data is not de-reddened. The accuracy in determining the flux is $10 \%$, which includes both calibration error and the actual measurement uncertainty. The velocities can be estimated to a precision of $\sim 1 / 2$ of a pixel, corresponding to about $25 \mathrm{~km} \mathrm{~s}^{-1}$.

\begin{tabular}{|c|c|c|c|c|c|c|c|}
\hline Ion & $\begin{array}{l}\lambda_{0} \\
(\AA)\end{array}$ & Feature & $\begin{array}{l}F W Z I \\
\left(\mathrm{~km} \mathrm{~s}^{-1}\right)\end{array}$ & $\begin{array}{l}\text { Peak vel. } \\
\left(\mathrm{km} \mathrm{s}^{-1}\right)\end{array}$ & $\begin{array}{l}F_{\text {tot }} \\
\left(10^{-15} \mathrm{erg} \mathrm{cm}^{-2} \mathrm{~s}^{-1}\right)\end{array}$ & $\begin{array}{l}\text { Max vel. } \\
\left(\mathrm{km} \mathrm{s}^{-1}\right)\end{array}$ & Comment \\
\hline \multirow[t]{4}{*}[\mathrm{NII}]{} & 6548.1 & $\mathrm{D}$ & 200 & -160 & 2.5 & -260 & \\
\hline & & $\mathrm{E}$ & - & -190 & 0.34 & - & \\
\hline & & $\mathrm{F}$ & 200 & -210 & 0.72 & -305 & \\
\hline & & $\mathrm{F} 2$ & 300 & - & 0.49 & -400 & \\
\hline \multirow[t]{5}{*}{$H \alpha$} & 6562.817 & $\mathrm{D}$ & 300 & -150 & 17.23 & -310 & \\
\hline & & $\mathrm{E}$ & 270 & -128 & 1.43 & -243 & \\
\hline & & $\mathrm{F}$ & 190 & -220 & 4.1 & -310 & \\
\hline & & $\mathrm{F} 2$ & 160 & -243 & 0.82 & -310 & \\
\hline & & $\mathrm{F} 3$ & 100 & -50 & 0.72 & -105 & \\
\hline \multirow[t]{4}{*}[\mathrm{NII}]{} & 6583.6 & $\mathrm{D}$ & 300 & -160 & 6.2 & -320 & \\
\hline & & $\mathrm{E}$ & - & - & - & - & \\
\hline & & $\mathrm{F}$ & 275 & -250 & 2.7 & -345 & \\
\hline & & $\mathrm{F} 2$ & 250 & -250 & 0.67 & -410 & \\
\hline \multirow[t]{5}{*}{ [SII] } & 6716.42 & $\mathrm{D}$ & 275 & -215 & 5.4 & -325 & $\Sigma_{\text {flux }(6717+6731)}=12.2^{a}$ \\
\hline & & $\mathrm{E}$ & - & - & - & - & \\
\hline & & $\mathrm{F}$ & 180 & -280 & 1.35 & -395 & $\Sigma_{\text {flux }(6717+6731)}=3.0^{a}$ \\
\hline & & $\mathrm{F} 2$ & 400 & - & 0.5 & -360 & $\Sigma_{\text {flux }(6717+6731)}=1.43^{a}$ \\
\hline & & F3 & 180 & -125 & 1.49 & -215 & $\Sigma_{\text {flux }(6717+6731)}=3.6^{a}$ \\
\hline \multirow[t]{5}{*}{ [SII] } & 6730.78 & $\mathrm{D}$ & 275 & -160 & 6.9 & -325 & \\
\hline & & $\mathrm{E}$ & 180 & -160 & 0.54 & -250 & \\
\hline & & $\mathrm{F}$ & 180 & -260 & 1.65 & -395 & \\
\hline & & $\mathrm{F} 2$ & 400 & -70 & 0.93 & -360 & \\
\hline & & F3 & 180 & -115 & 2.1 & -215 & \\
\hline \multirow[t]{3}{*}[\mathrm{FeII}]{} & 7155.14 & $\mathrm{D}$ & 210 & -170 & 2.4 & -270 & $F W H M=160 \mathrm{~km} \mathrm{~s}^{-1 b}$ \\
\hline & & $\mathrm{F}$ & 300 & -295 & 2.6 & -420 & $F W H M=140 \mathrm{~km} \mathrm{~s}^{-1}$ \\
\hline & & $\mathrm{F} 2$ & 250 & -330 & 0.78 & -430 & $F W H M=150 \mathrm{~km} \mathrm{~s}^{-1}$ \\
\hline [FeII] & 7172. & $\mathrm{D}$ & - & - & 0.33 & - & \\
\hline [CaII $]$ & 7291.46 & $\mathrm{D}$ & 250 & -165 & 6.1 & -290 & \\
\hline$[\mathrm{NiII}]$ & 7379.6 & $\mathrm{D}$ & - & - & 1.95 & - & $b$ \\
\hline$[\mathrm{FeII}]$ & 7452.54 & $\mathrm{D}$ & - & - & 0.86 & - & $b$ \\
\hline [FeII] & 8619.33 & $\mathrm{D}$ & 250 & -120 & 0.64 & -250 & $b$ \\
\hline
\end{tabular}

of the reverse shock or Mach disk $(H \alpha)$, and the forward, or bow-shock (SII). The bright $\mathrm{H} \alpha$ core of the working surface has a $F W H M$ of 0.3 arcsec at right angles to the major axis of the outflow, while the whole "working surface", knot D, has a $F W H M$ of $\sim 2$ arcsec. The separation between maximum [S II] (D4) and maximum $\mathrm{H} \alpha$ (D1) emission is 0.6 arcsec or about $2 \times 10^{15} \mathrm{~cm}$ deprojected for a viewing angle of $45^{\circ}$. We are thus resolving the structure of the shock.
This separation is consistent with the calculations of Hartigan (1989) that - given a cooling distance of $\sim R_{\text {jet }}$ (=the jet radius) - show that both the forward shock, i.e. the bowshock, and the Mach disk will be radiative and that the separation should be just equal to $\sim R_{\text {jet }}$, as is true in this case. In our data both the cooling length - i.e. the distance between D1 and D4 - and the jet radius appear to be of the same order $\left(\sim 10^{15} \mathrm{~cm}\right)$. According to the model of Hartigan (1989), a 
Table 5. Proper motions measured for features in the jet. Note that these data are obtained only from the HST 1996 and 1998 observations. The actual precision of measurement is 0.1 arcsec. Note that the velocity data for the F-complex is somewhat uncertain, since the identification of individual features (particularly $\mathrm{F}^{\prime}$ ) have some ambiguity.

\begin{tabular}{|c|c|c|c|c|c|c|}
\hline Feature & $\begin{array}{l}\mathrm{H} \alpha \\
\operatorname{arcsec}\end{array}$ & $\begin{array}{l}\text { [S II }] \\
\operatorname{arcsec}\end{array}$ & $\begin{array}{l}\text { Average } \\
\text { arcsec }\end{array}$ & $\begin{array}{l}\text { Velocity } \\
\operatorname{arcsec}_{\mathrm{yr}^{-1}}\end{array}$ & $\begin{array}{l}\text { Velocity@150 pc } \\
\mathrm{km} \mathrm{s}^{-1}\end{array}$ & $\overline{\text { Comment }}$ \\
\hline F2 & 0.76 & $0 ! 73$ & 0.745 & $0 ! 35$ & 250 & See text about F-complex \\
\hline F' & 0.6 & 0.7 & 0.65 & 0.3 & 220 & difficult to identify unambiguously \\
\hline $\mathrm{F}$ & 0.5 & 0.7 & 0.6 & $0 ! 3$ & 220 & See text about F-complex \\
\hline $\mathrm{E}$ & 0.53 & $0 ! 41$ & 0.47 & 0.22 & 160 & \\
\hline D2 & 0.65 & 0.62 & 0.635 & 0.30 & 215 & \\
\hline D1 & 0.44 & 0.45 & 0.445 & $0 ! 21$ & 150 & \\
\hline D4 & _ & 0.44 & $0 ! 44$ & 0.20 & 150 & \\
\hline D3 & $0 ! 36$ & - & $0 ! 36$ & $0 ! 17$ & 120 & \\
\hline
\end{tabular}

Table 6. The length of the jet at different epochs. $\Delta$ is the number of days between successive epochs. The measurement precision is $\sim 0.1$ arcsec.

\begin{tabular}{lllll}
\hline \hline Filter & Year & $\begin{array}{l}\Delta t \\
\text { days }\end{array}$ & $\begin{array}{l}\text { Length } \\
\operatorname{arcsec}\end{array}$ & $\begin{array}{l}\text { Rate of change } \\
\operatorname{arcsec} \mathrm{yr}^{-1}\end{array}$ \\
\hline$R$ & 1989 & 0 & $10^{\prime} 4$ & na \\
$R$ & 1993 & 1364 & $11^{\prime \prime} 0$ & 0.16 \\
$R$ & 1996 & 935 & $12^{\prime \prime} 24$ & 0.48 \\
$\mathrm{H} \alpha+[\mathrm{S} \mathrm{II}]$ & 1998 & 727 & $12^{\prime \prime} 73$ & 0.25 \\
$\mathrm{H} \alpha$ & 2001 & 1316 & $14^{\prime \prime} 32$ & 0.44 \\
\hline
\end{tabular}

Table 7. The change of distance of features A, B and C.

\begin{tabular}{|c|c|c|}
\hline Feature & 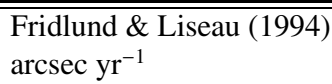 & $\begin{array}{l}1993-2001 \\
\operatorname{arcsec} \mathrm{yr}^{-1}\end{array}$ \\
\hline$\overline{\mathrm{A}}$ & $0 ! 29 \pm 0,06$ & $0 .^{\prime} 1 \pm 0 .{ }^{\prime} 1$ \\
\hline B & $0.26 \pm 0.06$ & $0 \prime^{\prime} 18 \pm 0 .{ }^{\prime} 1$ \\
\hline $\mathrm{C}$ & $0.26 \pm 0^{\prime} 05$ & $0.20 \pm 0 .{ }^{\prime} 1$ \\
\hline
\end{tabular}

Mach disk which is - as in this case - significantly brighter and here also with a much higher level of excitation than the bow-shock (see Fridlund et al. 1997; also Fig. 3) is indicative of a less dense jet penetrating into a denser ambient medium. It is at this interface where it is possible that the transfer of momentum from the fast atomic wind to the slower molecular outflow is taking place.

The model of Hartigan (1989) predicts the relative density of the jet and the ambient medium surrounding the jet in terms of the relative brightness of the bow-shock and the Mach disk, and the jet velocity. For the HH154 jet, we find the density of the jet:

$\rho_{\text {jet }} \sim(0.05-0.1) \times \rho_{\text {ambient }}$.

An analysis taking into account the $n_{\mathrm{e}}$ derived in the previous section and the molecular data of Fridlund \& Knee (1993) then shows that the ionisation fraction of the jet is $\sim 1$ (FL98). This led to the conclusion in that paper that the optical jet is not driving the molecular outflow, since there is a lack of the necessary momentum by at least 2 orders of magnitude. In FL98, however, we assumed a velocity of $300 \mathrm{~km} \mathrm{~s}^{-1}$ for the jet material (since we then did only have access to low dispersion spectroscopy). Inspection of Fig. 8, demonstrates that most of the
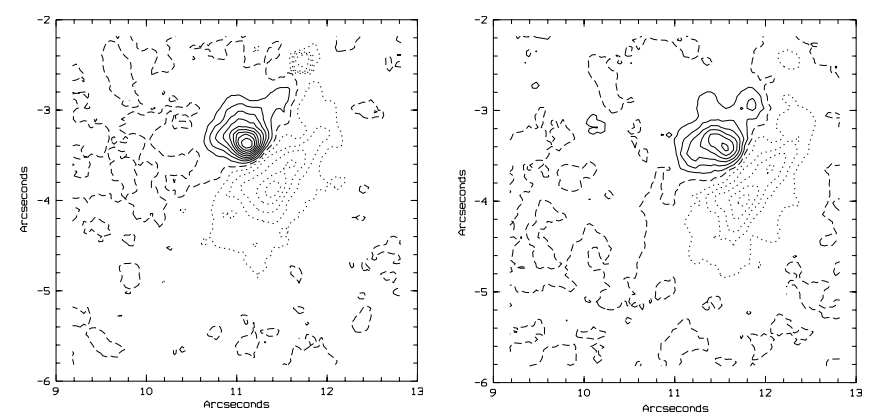

Fig. 7. Difference images of the working surface (feature D - see text) between the $\mathrm{H} \alpha$ and [S II] images in 1996 (top) and 1998 (bottom). The positions are relative to an arbitary position.

material is found at velocities less than this value. Some material is traveling at velocities close to $600 \mathrm{~km} \mathrm{~s}^{-1}$, when taking the inclination into account, but we note that even if we were to allow the bulk of the material to travel at this higher velocity, it would still leave us lacking at least one order of magnitude of momentum (something which would also require a $100 \%$ efficient mechanism for the transfer of the jet momentum to the molecular outflow). In this context, we also note that there are no obvious indications of so called "turbulent entrainment", and that further the interface for "prompt entrainment" (i.e. at the Mach disk) is very small - only 30 AU across. In this context, we wish to note, that the molecular momentum calculated by Fridlund \& Knee (1993) refers to the present outflow, i.e. with a dynamical time scale of less than $\sim$ a few hundred years which is therefore directly comparable to the jet dynamical time scale.

In the $\mathrm{H} \alpha$-[S II] difference images in Fig. 7 we see indications of a change of position of the Mach disk. If we assume that the location of this shock is the sharp interface between the [S II] and the $\mathrm{H} \alpha$ emission, we can see that it has moved $(0.5$ arcsec $)$ further out from IRS5 in $\sim 2$ years. This corresponds to a velocity of $\sim 180 \mathrm{~km} \mathrm{~s}^{-1}$, assuming a distance of $150 \mathrm{pc}$. There is also an indication that the actual apex of the shock is turning towards the north - something that could indicate rotation of the jet material. 

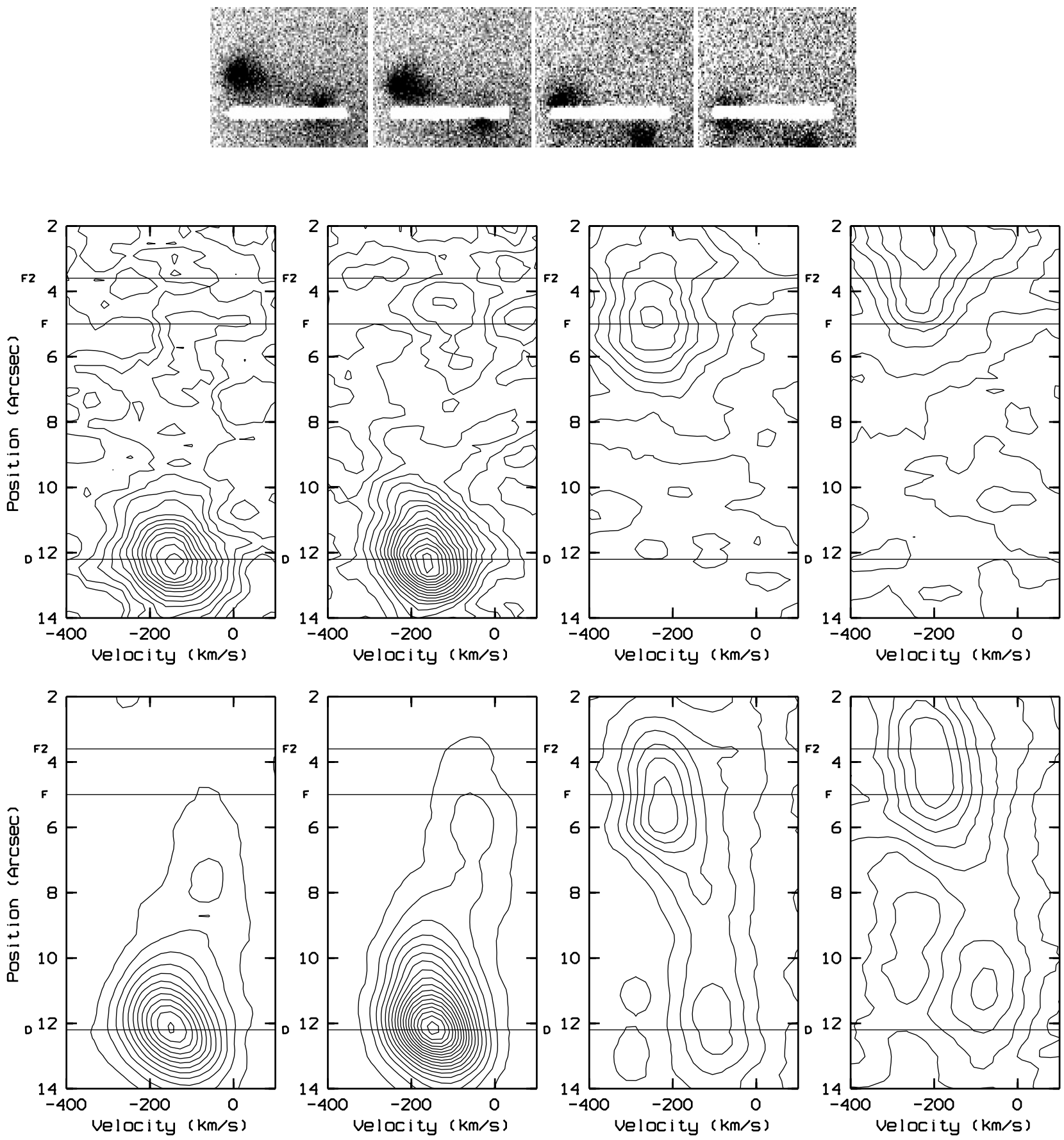

Fig. 8. The $\mathrm{H} \alpha$ and [Fe II] $7155 \AA$ spectra obtained with ALFOSC. In the top row, the different slit positions are shown superposed on $20 \mathrm{~s}$ $R$-band images. The effective slit width is $12^{\prime \prime}$ long. In the middle row are the [Fe II] spectra and in the bottom rows are the H $\alpha$ spectra. All are displayed as contour maps, where the contours have been selected to show the maximum extent of emission. A radial velocity scale wrt barycentric zero has been used. Comparing on the one hand the spectra of $\mathrm{H} \alpha$ and [Fe II] $7155 \AA$ spectra and on the other the slit positions with repsect to the jet, clearly demonstrate the two different velocity systems, originally discovered by FL98. At position $12.2^{\prime \prime}$, in both spectral lines, feature D is prominent. A peak velocity of $160 \mathrm{~km} \mathrm{~s}^{-1}$ is indicated. Features D, F and F2 have been marked according to their 1998 HST positions

\subsection{The location of the $X$-ray source}

Favata et al. (2002) discovered 1-2 keV X-rays emanating from the jet. Using XMM-Newton, the positional uncertainty included most of the jet. Nevertheless, these authors suggested that the X-rays were possibly emanating from the working surface in view of it being identified as a strong shock (FL98).
This was in spite of it having a lower radial velocity and proper motion (both $\sim 160 \mathrm{~km} \mathrm{~s}^{-1}$ ) than features found closer to IRS5 along the jet. Bally et al. (2003), using the Chandra X-ray observatory, have improved on the position, and indeed locate the X-ray source in the innermost part of the jet $(\sim 1$ arcsec from the radio position of IRS5). As can be seen from Fig. 8 in this paper, at this position we find radial velocities for features $\mathrm{F}$ 
and F2 of up to $430 \mathrm{~km} \mathrm{~s}^{-1}$, which given the assumed inclination of the jet of $\sim 45^{\circ}-\sim 60^{\circ}$ (Liseau et al. 2005) represent true velocities of $\sim 500 \mathrm{~km} \mathrm{~s}^{-1}$ to $\sim 600 \mathrm{~km} \mathrm{~s}^{-1}$. The immediate post-shock temperature is (Raga 1989):

$T_{\mathrm{ps}} \simeq \frac{2.9 \times 10^{5} \mathrm{~K}}{1+X} \times\left(\frac{v_{\text {shock }}}{100 \mathrm{~km} \mathrm{~s}^{-1}}\right)^{2}$.

Here $X$ is the hydrogen preionisation fraction and an abundance of $0.9(\mathrm{H})$ and $0.1(\mathrm{He})$ by number has been assumed. As mentioned before, FL98 have come to the conclusion, that it is very likely that the ionisation fraction is close to unity.

With these assumptions, we calculate a shock temperature of $\sim 3 \times 10^{6} \mathrm{~K}$ to $\sim 10^{7} \mathrm{~K}$, in excellent agreement with the X-ray results of Favata et al. (2002) and Bally et al. (2003). We also note here that the material detected at the highest radial velocities probably emanates from the recombination/cooling region "downstream" of the X-ray generating shock, where in all likelihood the velocity has dropped somewhat. All this information taken together therefore implies that the emission found at the highest radial velocities in our data, emanating from inside of the feature F2, is associated with the same location (or very close to) the source of the X-rays. Thus where the jet first becomes visible as it emerges from behind the obscuration. As can be seen from Table 2, the extinction toward feature F2 is compatible with the values determined from the XMM spectrum (Favata et al. 2002).

We note here that the presence of an X-ray source at a distance of $\sim 200 \mathrm{AU}$ from the surface of the disk, as well as other shock phenomena generating Lyman photons up to maybe $1000 \mathrm{AU}$ to $1500 \mathrm{AU}$ further away, are going to have an impact on the ionisation structure of the disk surface. This, in turn, could have an impact on models trying to explain the molecular outflows, such as MHD winds lifting off the disk surface.

\subsection{The bright feature in the inner region of the jet}

Both the two jets and the northern arc first appear in the same location in the sky ("apex") and have an opening angle of about $90^{\circ}$ (see Fig. 2 - the angle between the northern arc and the southern jet at their intersection). The jet emission is seen superposed onto a fan-shaped nebulosity that is most evident in the $I$-band filter. The northern arc and the southern jet then bend inwards towards the northern jet - the northern arc more drastically than the southern jet - and after 1.4 arcsec all 3 components are parallel. Note that it is within this envelope that X-ray emission is found to originate (Bally et al. 2003).

Absolute positional information in the HST data is restricted to an accuracy of $\sim 1$ arcsec, but our images are consistent with the radio position of IRS5 (Looney et al. 1997) being within 0.5 arcsec from the origin of the visible jet. Only 0.6 arcsec SW from the apex is a very bright feature (see Fig. 2 bottom) visible in the $I$-band. It is also faintly visible in the $R$-band image, but is actually the brightest feature in the $I$-band. This knot appears elliptical with dimensions of about 0.4 arcsec by $0.2 \operatorname{arcsec}(F W H M)$. A careful analysis shows it to consist of two components, with a separation of 0.145 arcsec or $\sim 20 \mathrm{AU}$ at the distance of L1551. An approximate $R-I$ colour for both sources is found to be $\sim+2.0 \mathrm{mag}$. The feature is located between the northern jet and the southern jet, and at an angle offset from the outflow direction of those two features. (White et al. 2000), have analysed near-infrared HST-NICMOS data covering continuum and emission lines between $1.12 \mu \mathrm{m}$ and $2.12 \mu \mathrm{m}$. Inspection of these frames confirm the interpretation here, since faint [Fe II] emission is found from knot F, while a strong continuum peak is visible at the same position as the oval feature described here. No molecular hydrogen emission is detected in the NICMOS data.

Since the jet emission is mostly caused by recombination presumably through interactions of the outflowing gas through shock phenomena, and since the oval feature described here is totally absent in the $\mathrm{H} \alpha$ and [S II] images we suggest that it is instead caused by light from the IRS5 system scattered up through a polar hole in the circumstellar disk and reflected off the inside of the "jet-cavity". Its dimensions of $~ 60 \mathrm{AU}$ by $30 \mathrm{AU}$, with two peaks separated by $\sim 20 \mathrm{AU}$, is also consistent with the binary nature and parameters of the IRS5 system. This is supported by the fact that Rodríguez et al. (2003a,b) have demonstrated that the jets can be traced in towards the binary behind the obscuration, and indeed it thus appears likely that they together excavate a "polar hole" through which the light from the stars/accretion disks can escape.

\section{Conclusions}

In summary, we find:

- We suggest that the bright, oval feature detected close to the apex of the jet in the $I$-band is caused by light from the IRS5 binary system scattered up through a (polar?) hole in the circumstellar disk and reflected off the inside of the "jet-cavity" towards our direction. This is supported by the colour of the feature.

- We find that the position of the origin of the X-rays emanating from the jet is coincident or very nearly so with the location of the observed maximum radial velocities velocities (representative of true velocities of $\sim 500 \mathrm{~km} \mathrm{~s}^{-1}$ $\left.600 \mathrm{~km} \mathrm{~s}^{-1}\right)$.

- We have mapped out the proper motions resulting in transverse velocities, as well as mapping the complete radial velocity field of the jet. A few jet features (e.g. knot E) now have very much different velocities. We suggest this is because the old feature has disappeared and we are actually observing a new "interstellar bullet" traveling down the jet towards the working surface. The time scale for the ejection of bullet like features is thus of the order of a few years.

- The average rate of change of the length of the whole jet, found during our observing period (1 Dec. 1989 to 21 Oct. $2001=4342$ days) is $0.3 \operatorname{arcsec} \mathrm{yr}^{-1}$ representative of a transverse velocity of $235 \mathrm{~km} \mathrm{~s}^{-1}$.

- The time scale for variations of physical conditions within or near the jet (like the excitation conditions or the extinction) is of the order of a few years. This is similar to the time scale for morphological changes.

- Within the F-complex of knots, we find a number of well defined moving features $\left(\mathrm{F}, \mathrm{F}^{\prime}\right.$ and $\left.\mathrm{F} 2\right)$, with large 
proper motions, but with much different excitation conditions among them.

Further, we also find:

- The excitation conditions and the morphology within the feature D identify it as a "working surface" where the jet impacts either the ambient medium or material moving at a slower speed (from a previous ejection event).

- In the latter context, it is noteworthy, that feature D has maintained its individual character during more than 20 years and while traveling more than 1000 AU further downstream.

- The Mach disk and the forward (or bow-)shocks are well resolved. The separation between high excitation (as traced by $\mathrm{H} \alpha$ ), and low-excitation (as traced by [S II] in the working surface (knot D) is $\sim 0.6$ arcsec.

- The Mach disk is moving "downstream" with a transverse velocity of $\sim 180 \mathrm{~km} \mathrm{~s}^{-1}$. During the time interval between our HST observations it also appears to have changed direction.

- The cooling distance in the working surface of the northern jet is $\sim$ equal to the jet radius, $R_{\text {jet }} \sim 0.6$ arcsec

Acknowledgements. We gratefully acknowledge valuable discussions with Drs. G. Olofsson, A. Heras, T. Prusti and G. Pilbratt. Some of the data presented here have been taken using ALFOSC, which is owned by the Instituto de Astrofisica de Andalucia (IAA) and operated at the Nordic Optical Telescope under agreement between IAA and the NBIfAFG of the Astronomical Observatory of Copenhagen. The comments and positve criticism of the referee, Dr. Bo Reipurth, which led to a more stringent paper, are gratefully acknowledged.

\section{References}

Bally, J., Feigelson, E., \& Reipurth, B. 2003, ApJ, 584, 843

Bieging, J. H., \& Cohen, M. 1985, ApJ, 289, L5

Campbell, B., Persson, S. E., Strom, S. E., \& Grasdalen, G. L. 1988, AJ, 95, 1173

Cardelli, J. A., \& Clayton, G. C. 1988, AJ, 95, 516

Cohen, M., \& Fuller, G. A. 1985, ApJ, 296, 620

Cohen, M., Bieging, J. H., \& Schwartz, P. R. 1982, ApJ, 253, 707

Cudworth, K. M., \& Herbig, G. 1979, AJ, 84, 548

Devine, D., Reipurth, B., \& Bally, J. 1999, AJ, 118, 972

Devine, D., Bally, J., Reipurth, B., Stocke, J., \& Morse, J. 2000, ApJ, 540, L57

Favata, F., Fridlund, C. V. M., Micela, G., Sciortino, S., \& Kaas, A. A. 2002, A\&A, 386, 204

Fridlund, C. V. M., \& Liseau, R. 1994, A\&A, 292, 631

Fridlund, C. V. M., \& Liseau, R. 1998, ApJ, 499, L75

Fridlund, C. V. M., Nordh, H. L., van Duinen, R. J., Aalders, J. W. G., \& Sargent, A. I. 1980, A\&A, 91, L1
Fridlund, C. V. M., Sandqvist, A., Nordh, H. L., \& Olofsson, G. 1984, A\&A, 137, L17

Fridlund, C. V. M., Liseau, R., \& Perryman, M. A. C. 1993, A\&A, 273, 601

Fridlund, C. V. M., Liseau, R., \& Gullbring, E. 1998, A\&A, 330, 327

Fridlund, C. V. M., Bergman, P., White, G. J., Pilbratt, G. L., \& Tauber, J. A. 2002, A\&A, 382, 573

Fridlund, C. V. M., Djupvik, A. A., Liseau, R., et al. 2005, in preparation

Fridlund, M., Huldtgren, M., \& Liseau, R. 1997, in Herbig-Haro Flows and the Birth of Stars, IAU Symp., 182, 19

Hartigan, P. 1989, ApJ, 339, 987

Hartigan, P., Raymond, J., \& Hartmann, L. 1987, ApJ, 316, 323

Heathcote, S., Morse, J. A., Hartigan, P., et al. 1996, AJ, 112, 1141

Holtzman, J. A., Burrows, C. J., Casertano, S., et al. 1995, PASP, 107, 1065

Keene, J., \& Masson, C. R. 1990, ApJ, 355, 635

Kenyon, S. J., Dobrzycka, D., \& Hartmann, L. 1994, AJ, 108, 1872

Ladd, E. F., Fuller, G. A., Padman, R., Myers, P. C., \& Adams, F. C. 1995, ApJ, 439, 771

Liseau, R., Fridlund, C. V. M., \& Larsson, B. 2005, ApJ, 619, 959

Looney, L. W., Mundy, L. G., \& Welch, W. J. 1997, ApJ, 484, L157

Mundt, R., \& Fried, J. W. 1983, ApJ, 274, L83

Mundt, R., Ray, T. P., \& Raga, A. C. 1991, A\&A, 252, 740

Neckel, T., \& Staude, H. J. 1987, ApJ, 322, L27

Norman, C., \& Silk, J. 1979, ApJ, 228, 197

Pyo, T., Hayashi, M., Kobayashi, N., et al. 2002, ApJ, 570, 724

Pyo, T., Hayashi, M., Kobayashi, N., et al. 2005, ApJ, 618, 817

Quinet, P., Le Dourneuf, M., \& Zeippen, C. J. 1996, A\&AS, 120, 361

Raga, A. C. 1989, in Low Mass Star Formation and Premain Sequence Objects, ed. B. Reipurth, European Southern Observatory, Garching bei Munchen, 281

Raga, A. C., Böhm, K. H., \& Cantó, J. 1996, Rev. Mex. Astron. Astrofis., 32, 161

Raga, A. C., Riera, A., Masciadri, E., et al. 2004, AJ, 127, 1081

Reipurth, B. 1999, A general catalogue of Herbig-Haro objects, electronically published via World Wide Web at

http://casa.colorado.edu/hhcat

Reipurth, B., Hartigan, P., Heathcote, S., Morse, J. A., \& Bally, J. 1997, AJ, 114, 757

Rieke, G. H., \& Lebofsky, M. J. 1985, ApJ, 288, 618

Rodríguez, L. F., Canto, J., Torrelles, J. M., \& Ho, P. T. P. 1986, ApJ, 301, L25

Rodríguez, L. F., Curiel, S., Cantó, J., et al. 2003a, ApJ, 583, 330

Rodríguez, L. F., Porras, A., Claussen, M. J., et al. 2003b, ApJ, 586, L137

Sarcander, M., Neckel, T., \& Elsaesser, H. 1985, ApJ, 288, L51

Snell, R. L., Bally, J., Strom, S. E., \& Strom, K. M. 1985, ApJ, 290, 587

Snell, R. L., Loren, R. B., \& Plambeck, R. L. 1980, ApJ, 239, L17

Solf, J., Bohm, K. H., \& Raga, A. 1988, ApJ, 334, 229

White, G. J., Liseau, R., Men'shchikov, A. B., et al. 2000, A\&A, 364, 741 